\title{
A Fast Algorithm for Optimally Finding Partially Disjoint Shortest Paths
}

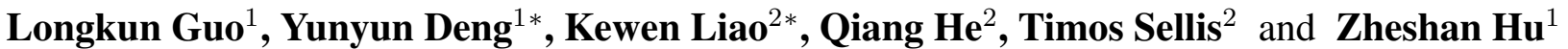 \\ ${ }^{1}$ College of Mathematics and Computer Science, Fuzhou University, Fuzhou 350116, Fujian, China \\ ${ }^{2}$ Department of Computer Science and Software Engineering, Swinburne University, VIC, Australia \\ lkguo@fzu.edu.cn, \{kliao, qhe, tsellis\}@ swin.edu.au
}

\begin{abstract}
The classical disjoint shortest path problem has recently recalled interests from researchers in the network planning and optimization community. However, the requirement of the shortest paths being completely vertex or edge disjoint might be too restrictive and demands much more resources in a network. Partially disjoint shortest paths, in which a bounded number of shared vertices or edges is allowed, balance between degree of disjointness and occupied network resources. In this paper, we consider the problem of finding $\mathrm{k}$ shortest paths which are edge disjoint but partially vertex disjoint. For a pair of distinct vertices in a network graph, the problem aims to optimally find $\mathrm{k}$ edge disjoint shortest paths among which at most a bounded number of vertices are shared by at least two paths. In particular, we present novel techniques for exactly solving the problem with a runtime that significantly improves the current best result. The proposed algorithm is also validated by computer experiments on both synthetic and real networks which demonstrate its superior efficiency of up to three orders of magnitude faster than the state of the art.
\end{abstract}

\section{Introduction}

Several shortest path problems [Akiba et al., 2015; Hansen and Abdoulahi, 2016; Imamichi et al., 2016] have recently witnessed renewed interests in AI community. Solutions to these problems are largely heuristic-based. In network planning and optimization, the classical disjoint shortest path problem, especially the problem of finding partially disjoint shortest paths, has also started with a heuristic method [Seymour and Kar, 2013] and only recently seen guaranteed optimal solutions [Yallouz et al., 2016]. However, the state-ofart optimal solutions are far from being efficient and scalable, limiting their practicability. In this paper, we investigate a fast solution to the following problem of finding $k$ edge-disjoint shortest paths with a bounded number of common vertices:

\footnotetext{
${ }^{*}$ These two authors share equal contribution.
}

Definition 1. (The $\delta$-vertex $k$ edge-disjoint shortest path problem, $\delta V-k E D S P)$ : For a directed graph or network $G=$ $(V, E)$ with a source vertex $s \in V$ and a destination $t \in V$, a weight function $w: E \rightarrow Z^{+}$, a given nonnegative integer $\delta \in Z, \delta V-k E D S P$ aims to compute $k$ edge-disjoint paths $P_{1}, P_{2}, \cdots, P_{k}$ in $G$, such that $\sum_{i=1, \cdots, k} w\left(P_{i}\right)$ attains the minimum while among them there are at most $\delta$ vertices (besides $s$ and $t$ ) shared by at least two paths.

Intuitively, the above definition constrains edge-disjoint shortest paths intersecting each other with the number of intersection (node) points upper bounded by $\delta$. Compared with the other stricter vertex-disjoint shortest paths problem, $\delta V$ $k E D S P$ potentially finds a set of paths with a shorter average length. For the problem when $k=2$, we propose a fast optimal (or namely exact) algorithm to $\delta V-2 E D S P$, as we can already envision that the general $\delta V-k E D S P$ requires much more algorithmic machinery. Note that throughout the paper we use terms "shortest" and "minimum weight", and also "exact algorithm" and "optimal algorithm", interchangeably.

\subsection{Related Work}

To the best of our knowledge, the $\delta V$-kEDSP problem was first studied in [Yallouz et al., 2016] for $k=2$, where it was optimally solved within a time complexity $O\left(m n^{2}+n^{3} \log n\right)$. Although the study over $\delta V$ - $k E D S P$ is emerging, the case of $\delta=0$ has already attracted intensive research from mathematicians and computer scientists starting from early $70 \mathrm{~s}$. When $\delta=0, \delta V$ - $k E D S P$ becomes the complete vertex-disjoint (edge-disjoint) shortest $s t$-path problem, namely the min-sum vertex-disjoint path problem, which can be simply solved with a simple max flow. It was also shown by Suurballe that the problem, for any fixed integer $k>0$, admits an optimal algorithm with a runtime $O\left(n^{2} \log n\right)$ [Suurballe, 1974]. Later in [Suurballe and Tarjan, 1984], this runtime was improved to $O\left(m \log _{(1+m / n)} n\right)$.

Another important variant of $\delta V-k E D S P$ is the NodeDisjoint Path with Congestion $(N D P w C)$ problem. The problem is to maximize the number of paths to respectively connect $k$ given pairs of vertices $s_{1} t_{1}, \ldots, s_{i} t_{i}, \ldots, s_{k} t_{k}$, such that each vertex appears on at most $\gamma$ paths for a given positive integer $\gamma$ that is known as congestion factor [Chekuri et al., 2005; Chekuri and Ene, 2013]. The existing methods mostly modeled and relaxed the $N D P w C$ problem as 
a natural multicommodity flow problem via linear programming. Chekuri et al [Chekuri et al., 2005] used the multicommodity flow LP-relaxation to obtain an $O\left(\log ^{2} k \log n\right)$ approximation with congestion $\gamma=4$ for $N D P w C$ in planar graph. Then, Chekuri and Ene [Chekuri and Ene, 2013] gave a polynomial time algorithm which routes $\Omega\left(\frac{O P T}{\text { poly } \log k}\right)$ pairs with $O(1)$ congestion in undirected graphs, where $O P T$ is the value of an optimal fractional solution to a natural multicommodity flow relaxation. For the edge-disjoint case, the edge-disjoint path with congestion $(E D P w C)$ problem is also to maximize the number of disjoint paths respectively connecting the given pairs of vertices, such that each edge appears on at most $\gamma$ paths. For the first time addressing $E D P w C$, Raghavan and Thompson [Raghavan and Tompson, 1987] gave a constant factor approximation with congestion $\gamma$ relaxed to $\Omega(\log n / \log \log n)$ by using randomized rounding technique. Later, Andrew presented a randomized $\left(O\left(\log ^{61} n\right), O\left((\log \log n)^{6}\right)\right)$-approximation algorithm for $E D P w C$ in [Andrews, 2010]. Note that for inapproximability, Andrews et al. [Andrews et al., 2005] have also shown that for any congestion $\gamma=O\left(\frac{\log \log n}{\log \log \log n}\right)$, there is no $\log { }^{\frac{1-\epsilon}{\gamma+1}} n$-approximation algorithm for EDPwC unless $N P \subseteq Z P T I M E\left(n^{\text {poly } \log n}\right)$, so their poly-logarithmic bifactor ratio is nearly the best possible. For better congestion guarantee but fewer paths between pairs of vertices, Chuzhoy in [Chuzhoy, 2016] recently gave an efficient randomized algorithm, which routes $\Omega\left(\frac{O P T}{\log ^{22.5} k \log \log k}\right)$ demand pairs with congestion of at most 14 .

Our techniques for solving $\delta V-k E D S P$ involve at the last step a transformation to the restricted shortest path (RSP) problem that is known $\mathcal{N} \mathcal{P}$-complete in [Garey and Johnson, 2002]. To the best of our knowledge, Joksch [Joksch, 1966] was the first to formally solve RSP by presenting a pseudo-polynomial time algorithm using dynamic programming. Although we adopt this pseudo-polynomial algorithm for $R S P$ to form our polynomial time solution (this works because in our case $\delta \leq n$ ), it is worthwhile to mention other related approximations: Hassin gave another pseudo-polynomial algorithm with runtime $O(m O P T)$ for $O P T$ being the weight of an optimal solution, and then consequently gave an FPTAS with time $O\left(m\left(\frac{n^{2}}{\epsilon}\right) \log \left(\frac{n}{\epsilon}\right)\right)$ [Hassin, 1992]; While Lorena and Raz [Lorenz and Raz, 2001] proposed another FPTAS with a further improved runtime $O\left(m n\left(\log \log n+\frac{1}{\epsilon}\right)\right)$, for any fixed real number $\epsilon>$ 0 . Recently, the research interest is also on the $k$-edge (vertex) disjoint restricted shortest path $(k R S P)$ problem which combines RSP and disjoint paths [Orda and Sprintson, 2004; Guo et al., 2015].

\subsection{Our Results}

In this paper, we propose an exact algorithm for $\delta V$-2EDSP with a runtime $O(\delta m+n \log n)$, which significantly improves the runtime $O\left(m n^{2}+n^{3} \log n\right)$ of the previous best solution [Yallouz et al., 2016]. Our algorithm is based on the main observation that, if $\delta V-2 E D S P$ is feasible then there must exist a special optimal solution, what we called a spiral optimal solution. The algorithm progresses with the idea of path aug- mentation. It first computes a shortest $s t$-path, then this path gets augmented to a pair of spiral st-paths via our specially constructed residual graph with an extra cost function to capture the number of shared vertices. Moreover, we show that if $\delta V-2 E D S P$ is feasible, then there must exist a spiral optimal solution whose shared vertices are all on the shortest $s t$-path. With techniques from graph theory and network flow theory, we prove that our algorithm produces an optimal solution to $\delta V-2 E D S P$. The proposed algorithm has the potential to extend to $\delta V-k E D S P$ for general $k$, but we omit this discussion here due to space limit. We also run experiments on both synthetic and real networks to validate the practical performance of our algorithm against other baselines. Experimental results show an up to three orders of magnitude runtime reduction matching our theoretical runtime analysis.

\section{Exact Algorithms for $\delta V-2 E D S P$}

In this section, we first focus on an important property of $\delta V$ $2 E D S P$ : there must exist a special optimal solution called spiral optimal solution if an instance of $\delta V-2 E D S P$ is feasible. Then we give an algorithm to compute such a spiral optimal solution, which equivalently solves $\delta V$-2EDSP.

\subsection{Spiral Optimal Solutions to $\delta V-2 E D S P$}

We denote a network by a digraph $G=(V, E)$, where $V$ and $E$ are respectively the sets of vertices and edges. Assume that $n=|V|$ and $m=|E|$. Given an $s t$-path $P$ and two vertices $u, v \in P$, we denote by $P(u, v)$ the subpath of $P$ from $u$ to $v$, and say $u \prec_{P} v$ if and only if $v \neq u$ appears on $P(u, t)$, i.e. $v \in P(u, t)$. For two paths $P_{1}$ and $P_{2}$, we say $P_{1}(u, v)$ is a maximal segment within $P_{1} \cap P_{2}$ iff (1) $P_{1}(u, v) \subseteq P_{1} \cap P_{2}$ and (2) there exists in $P_{1} \cap P_{2}$ no other segment that contains $P_{1}(u, v)$, that is, there exists no $P_{i}(x, y) \subseteq P_{1} \cap P_{2}, i \in$ $\{1,2\}$, such that $P_{i}(x, y) \supset P_{1}(u, v)$ holds.

Our algorithm will actually compute a special optimal solution called a spiral optimal solution, which is formally as follows (An example comparing a spiral optimal solution vs a non-spiral optimal solution is as depicted in Figure 1):

Definition 2. Let $P^{*}$ be a shortest st-path in $G$. Then an optimal solution to $\delta V-2 E D S P$, say $\mathcal{P}=\left\{P_{1}^{*}, P_{2}^{*}\right\}$, is a spiral optimal solution if the following two conditions both hold: (1) For any $u, v \in P^{*} \cap P_{i}^{*}, i \in\{1,2\}, u \prec_{P^{*}} v$ iff $u \prec_{P_{i}^{*}} v$; (2) Let $\mathcal{S}_{i}=P^{*} \cap P_{i}^{*}, i \in\{1,2\}$, be the set of maximal segments at which $P^{*}$ intersects with $P_{i}^{*}$. Then for any $s e g_{j}$, seg $g_{j^{\prime}} \in$ $\mathcal{S}_{i}, i \in\{1,2\}$, if seg s $_{j} \prec_{P_{i}^{*}}$ seg $_{j^{\prime}}$ then there must exist seg $\in$ $\mathcal{S}_{3-i}$, such that $\operatorname{seg}_{j} \prec_{P^{*}}$ seg $\prec_{P^{*}}$ seg $g_{j^{\prime}}$ holds on $P^{*}$.

Note that $P_{1}^{*}$ and $P_{2}^{*}$ in the above definition must be edgedisjoint and hence can only share common vertices.

We say $v_{j}, v_{j^{\prime}} \in P^{*} \cap P_{i}^{*}, i \in\{1,2\}$ are properly ordered, if $v_{j}, v_{j^{\prime}}$ satisfy Condition 1 ; Otherwise, say $v_{j} \prec v_{j^{\prime}}$ on $P^{*}$ while $v_{j^{\prime}} \prec v_{j}$ on $P_{i}^{*}, v_{j}$ and $v_{j^{\prime}}$ is antiordered. Note that there may exist $P_{i}^{*} \in \mathcal{P}, i \in\{1,2\}$, such that $P^{*}=P_{i}^{*}$.

The key idea of our algorithm is inspired by the Simplex method of solving linear programs, in which a special optimal solution called basic optimal solution, rather than any optimal solution, is computed. So similar to the correctness proof of the Simplex method, we need first to show it suffices 


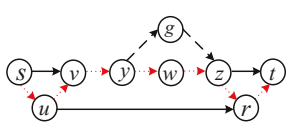

(a)

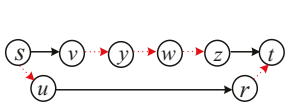

(b)

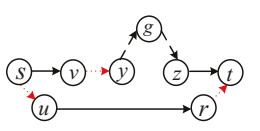

(c) $\rightarrow$ Weight 1 edge $\longrightarrow$ Weight 5 edge

Figure 1: A spiral optimal solution vs a non-spiral optimal solution. (a) An original graph with the shortest path $P^{*}$ (red edges); (b) A spiral optimal solution svywzt and surt; (c) A non-spiral optimal solution surt and svygzt.

to compute a spiral optimal solution to solve $\delta V-2 E D S P$, as given in the following theorem:

Theorem 3. If an instance of $\delta V-2 E D S P$ is feasible, then it must have spiral optimal solutions.

Proof. For Condition 1 of Definition 2, we show that if an instance of $\delta V-2 E D S P$ is feasible, then an optimal solution can be constructed satisfying the condition. Assume $\left\{P_{1}^{*}, P_{2}^{*}\right\}$ is an optimal solution of $\delta V-2 E D S P$, for which there exist $v_{j}, v_{j^{\prime}} \in P^{*} \cap P_{i}^{*}, i \in\{1,2\}$, such that $v_{j} \prec v_{j^{\prime}}$ on $P^{*}$ but $v_{j^{\prime}} \prec v_{j}$ on $P_{i}^{*}$. Then we can construct a pair of disjoint path $P_{1}$ and $P_{2}$ of which the number of antiordered vertex pairs decreases at least one comparing to $\left\{P_{1}^{*}, P_{2}^{*}\right\}$. Consequently, an optimal solution without any antiordered vertex pair can be constructed by repeatedly decreasing the number of antiordered vertex pairs.

It remains to give the construction of $P_{1}$ and $P_{2}$. Without loss of generality, we assume that $v_{j^{\prime}}$ is the vertex closest to $t$ in all antiordered vertex pairs. Let $u$, which belongs to $P_{i} \cup P_{3-i}$, be the first vertex after $v_{j^{\prime}}$. We will construct $P_{1}$ and $P_{2}$ for both two cases that $u \in P_{i}^{*}$ or $u \in P_{3-i}^{*}$ :

(1) $u \in P_{3-i}^{*}$ : Let $w \in P_{3-i}^{*} \cap P^{*}$ be the last vertex before $v_{j}$. We will show that there exists a solution $P_{1}$ and $P_{2}$ whose weight is not larger than that of $P_{1}^{*}$ and $P_{2}^{*}$, but with fewer antiordered vertex pairs. We need only to simply set $P_{1}=P_{i}^{*}\left(s, v_{j^{\prime}}\right) \cup P^{*}\left(v_{j^{\prime}}, u\right) \cup P_{3-i}^{*}(u, t)$ and $P_{2}=P_{3-i}^{*}(s, w) \cup P^{*}\left(w, v_{j}\right) \cup P_{i}^{*}\left(v_{j}, t\right)$. Apparently, every properly ordered vertex pair in $\left\{P_{1}^{*}, P_{2}^{*}\right\}$ remains so while the antiordered vertex pair $\left\{v_{j}, v_{j^{\prime}}\right\}$ becomes also properly ordered in $P_{1}$ and $P_{2}$. That is, the number of antiordered vertex pairs decreases at least one from $\left\{P_{1}^{*}, P_{2}^{*}\right\}$ to $\left\{P_{1}, P_{2}\right\}$. It remains only to show the weight of $\left\{P_{1}, P_{2}\right\}$ is not larger than $\left\{P_{1}^{*}, P_{2}^{*}\right\}$, which is to show $w\left(P^{*}\left(v_{j^{\prime}}, u\right) \cup P^{*}\left(w, v_{j}\right)\right) \leq w\left(P_{i}^{*}\left(v_{j^{\prime}}, v_{j}\right) \cup P_{3-i}^{*}(w, u)\right)$. Suppose otherwise, i.e., $w\left(P^{*}\left(v_{j^{\prime}}, u\right) \cup P^{*}\left(w, v_{j}\right)\right)>$ $w\left(P_{i}^{*}\left(v_{j^{\prime}}, v_{j}\right), P_{3-i}^{*}(w, u)\right) \geq w\left(P_{3-i}^{*}(w, u)\right)$. Then $P=$ $P^{*}(s, w) \cup P_{3-j}^{*}(w, u) \cup P^{*}(u, t)$ is an $s t$-path with a smaller weight than $P^{*}$, contradicting with the optimality of $P^{*}$. Therefore, the two constructed paths $P_{1}$ and $P_{2}$ are with a weight that is not larger than $\left\{P_{1}^{*}, P_{2}^{*}\right\}$.

(2) $u \in P_{i}^{*}$ : We will construct a new path $P_{i}$, which is with a weight no larger than $P_{i}^{*}$ but at least one less antiordered vertex pair. For the task, we need only to simply set $P_{i}=P_{i}^{*}\left(s, v_{j^{\prime}}\right) \cup P^{*}\left(v_{j^{\prime}}, u\right) \cup P_{i}^{*}(u, t)$. Apparently, $w\left(P_{i}\right) \leq w\left(P_{i}^{*}\right)$, because $w\left(P^{*}\left(v_{j^{\prime}}, u\right)\right) \leq w\left(P_{i}^{*}\left(v_{j^{\prime}}, v_{j}\right) \cup\right.$ $\left.P_{i}^{*}\left(v_{j}, u\right)\right)$ holds from the optimality of $P^{*}$. Besides, since $v_{j} \notin P_{i}, P_{i}$ is with at least one less antiordered vertex pair

than $P_{i}^{*}$. Moreover, according to the construction of $P_{i}$, the path pair $\left\{P_{i}, P_{3-i}^{*}\right\}$ shares only common vertices of the original $\left\{P_{i}^{*}, P_{3-i}^{*}\right\}$, and hence $\left\{P_{i}, P_{3-i}^{*}\right\}$ shares at most the same number of common vertices as $\left\{P_{i}^{*}, P_{3-i}^{*}\right\}$ which is bounded by $\delta$.

For Condition 2, let $\left\{P_{1}^{*}, P_{2}^{*}\right\}$ be an optimal solution to $\delta V-2 E D S P$ that already satisfies Condition 1 . Then we show an optimal solution also satisfying Condition 2 can be constructed from $\left\{P_{1}^{*}, P_{2}^{*}\right\}$. Let $s e g_{j}$ and $s e g_{j^{\prime}}$ be two segments in which all the interior vertices belong to $S_{1}=\{$ seg $\mid$ seg $\subseteq$ $\left.P_{1}^{*} \cap P^{*}\right\}$. Let $u$ be the last vertex in $\operatorname{seg}_{j}$ and $v$ be the first vertex in $\operatorname{seg}_{j^{\prime}}$. Assume that there exists no segment of $S_{2}=$ $\left\{\right.$ seg $\mid$ seg $\left.\subseteq P_{1}^{*} \cap P^{*}\right\}$ that appears on $P^{*}$ between $s e g_{j}$ and $s e g_{j^{\prime}}$. Then let $P_{1}=P_{1}^{*}(s, u) \cup P^{*}(u, v) \cup P_{1}^{*}(v, t)$, which is $P_{1}^{*}$ excepting replacing $P_{1}^{*}(u, v)$, the subpaths of $P_{1}^{*}$ between $s e g_{j}$ and $s e g_{j^{\prime}}$, by $P^{*}(u, v)$. Apparently, $P^{*}(u, v)$ is with a weight no larger than $P_{1}^{*}(u, v)$, so $w\left(P_{1}\right) \leq w\left(P_{1}^{*}\right)$. Moreover, seg $_{j}$ and $s e g_{j^{\prime}}$ are merged into one segment on $P_{1}$. Besides, $P_{1}$ and $P_{2}^{*}$ share only common vertices of $P_{1}^{*}$ and $P_{2}^{*}$. Therefore, by repeating such operations, clearly such a $\left\{P_{1}^{*}, P_{2}^{*}\right\}$ can eventually become a solution to $\delta V-2 E D S P$ satisfying both Condition 1 and Condition 2 .

Note that, Condition 2 will not hold trivially without Condition 1. Because in that case we could not simply use $P^{*}(u, v)$ to connect $s e g_{j}$ and $s e g_{j^{\prime}}$, as $s e g_{j^{\prime}} \prec_{P_{1}^{*}} \operatorname{seg}_{j^{\prime}}$ may hold. That is why we prove Condition 1 before Condition 2 . Moreover, because $P_{1}^{*}$ and $P_{2}^{*}$, as a spiral optimal solution to $\delta V$-2EDSP, are allowed to share $\delta$ common vertices, we have further observation on the relationship between their common vertices and $P^{*}$ as below:

Lemma 4. If an instance of $\delta V-2 E D S P$ is feasible, there must exist a spiral optimal solution $P_{1}^{*}$ and $P_{2}^{*}$ wrt $P^{*}$, such that the common vertices of $P_{1}^{*}$ and $P_{2}^{*}$ are all on $P^{*}$, i.e. $\left(V\left(P_{1}^{*}\right) \cap V\left(P_{2}^{*}\right)\right) \subseteq P^{*}$.

Proof. Suppose otherwise, then there exists a common vertex of $P_{1}^{*}$ and $P_{2}^{*}$ that does not belong to $P^{*}$, say $p \in P_{1}^{*} \cap P_{2}^{*}$ that $p \notin P^{*}$ holds. We will show that there exists a solution $P_{1}$ and $P_{2}$ whose weight is not larger than $P_{1}^{*}$ and $P_{2}^{*}$ but shares one less common vertex. Let $w, z \in P^{*} \cap P_{1}^{*}$ and $u, v \in P^{*} \cap P_{2}^{*}$ be the two pairs of vertices nearest to $p$ on $P_{1}^{*}$ and $P_{2}^{*}$, respectively. W.l.o.g., we assume $w \prec z$ on $P_{1}^{*}$ and $u \prec v$ on $P_{2}^{*}$, then $P_{1}^{*}$ and $P_{2}^{*}$ are as follows: $P_{1}^{*}=s \cdots \rightarrow w \cdots \rightarrow p \cdot \rightarrow \rightarrow z \cdots \rightarrow t$ and $P_{2}^{*}=s \cdots \rightarrow$ $u \cdots \rightarrow p \cdots \rightarrow v \cdots \rightarrow t$.

Following Condition 1 of Definition 2, there are six different permutations of $u, v, w$ and $z$ appearing on $P^{*}$ : (1) $w \prec u \prec v \prec z ;(2) w \prec u \prec z \prec v$; (3) $w \prec z \prec u \prec v$; (4) $u \prec w \prec v \prec z$; (5) $u \prec w \prec z \prec v$; (6) $u \prec v \prec w \prec z$. We need only to consider Case (1)-(3), since Case (4)-(6) are symmetrically similar. In the following, we will show in Case (1), $P_{1}^{*}$ and $P_{2}^{*}$ can not be a spiral optimal solution; while in Case (2) and (3), the number of common vertices decreases at least one:

(1) $w \prec u \prec v \prec z$ on $P^{*}$ : By Condition 2 of the definition of a spiral optimal solution, there must be at least a vertex $x \in$ $P_{1}^{*}$ appearing on $P^{*}$ between $u$ and $v$, i.e. $x \in P^{*}(u, v) \backslash$ $\{u, v\}$. Then because $w$ and $z$ are two vertices of $P^{*} \cap P_{1}^{*}$ that 


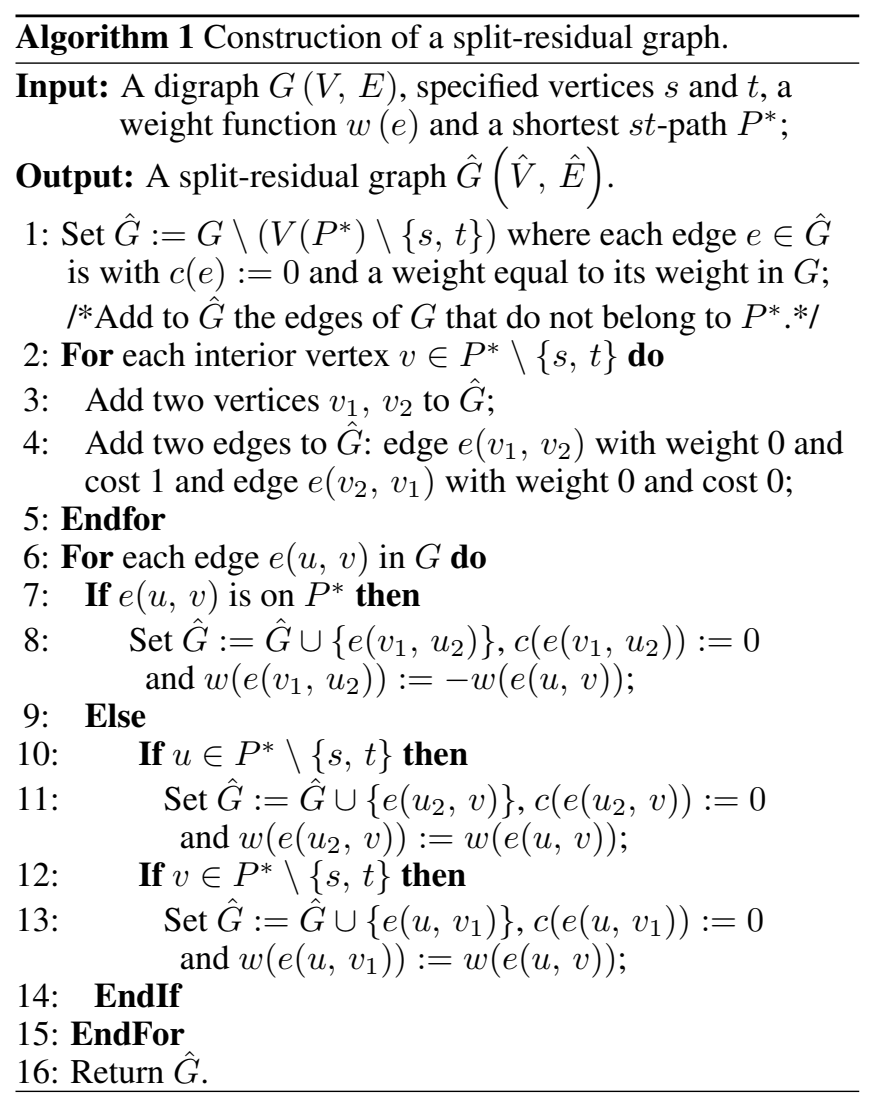

are closest to $p, x$ can only belong to $P_{1}^{*}(s, w)$ or $P_{1}^{*}(z, t)$. However, there will be an antiordered vertex pair $(w, x)$ for $x \in P_{1}^{*}(s, w)$ and $(z, x)$ for $x \in P_{1}^{*}(z, t)$. This contradicts with the fact that $\left(P_{1}^{*}, P_{2}^{*}\right)$ is a spiral optimal solution.

(2) $w \prec u \prec z \prec v$ on $P^{*}$ : Let $P_{1}=P_{1}^{*}(s, w, p) \cup$ $P_{2}^{*}(p, v, t)$ and $P_{2}=P_{2}^{*}(s, u) \cup P^{*}(u, z) \cup P_{1}^{*}(z, t)$. Obviously, $P_{1}$ and $P_{2}$ are two edge disjoint $s t$-paths with weight no larger than $P_{1}^{*}$ and $P_{2}^{*}$ and at least one less common vertex, i.e. no new common vertex emerges for $\left\{P_{1}, P_{2}\right\}$ and $p$ is no longer a common vertex.

(3) $w \prec z \prec u \prec v$ on $P^{*}$ : By Condition 2 of the definition of spiral optimal solution, there must be a vertex $x \in P_{1}^{*}$ appearing on $P^{*}(u, v) \backslash\{u, v\}$ while a vertex $y \in P_{2}^{*}$ appearing on $P^{*}(w, z) \backslash\{w, z\}$. Then let $P_{1}=P_{1}^{*}(s, w, p) \cup P_{2}^{*}(p, v, t)$ and $P_{2}=P_{2}^{*}(s, y) \cup$ $P^{*}(y, z, u, x) \cup P_{1}^{*}(x, t)$. Because $w\left(P^{*}(y, z, u, x)\right) \leq$ $w\left(P_{2}^{*}(y, u, p)\right)+w\left(P_{1}^{*}(p, z, x)\right)$ from the optimality of $P^{*}$, we have $w\left(P_{1}\right)+w\left(P_{2}\right) \leq w\left(P_{1}^{*}\right)+w\left(P_{2}^{*}\right)$. Moreover, $\left\{P_{1}, P_{2}\right\}$ has one less common vertex than $\left\{P_{1}^{*}, P_{2}^{*}\right\}$, since $p \notin P_{2}$ while no new common vertex is generated therein.

\subsection{The Exact Algorithm}

The key idea of our algorithm is inspired by the augmenting path algorithm for the max-flow problem [Ahuja et al., 1993]. Our algorithm will use a modified version of residual graph, namely split-residual graph, instead of traditional residual graph, but following the framework of the augmenting path algorithm: First, compute a shortest path, say $P^{*}$, and ac-

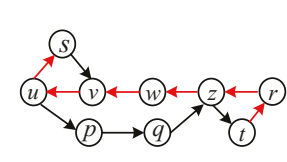

$\longrightarrow$ Reversed shortest path

(a)

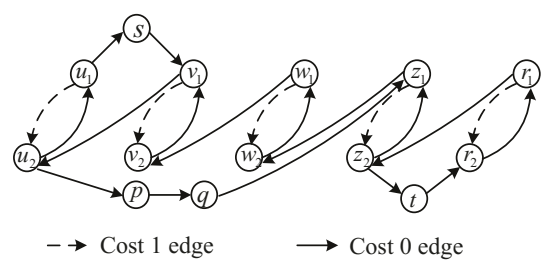

(b)
Figure 2: An example of executing Algorithm 1: (a) A residual graph with the reversed shortest path; (b) The split-residual graph.

cordingly construct a split-residual graph $\hat{G}$; Then compute a shortest $s t$-path in $\hat{G}$ to augment $P^{*}$, such that a spiral optimal solution to $\delta V-2 E D S P$ can be eventually obtained.

The difficulty of the algorithm is mainly in the construction of a split-residual graph $\hat{G}$ with respect to $G$ and $P^{*}$, such that $P^{*} \oplus Q$ is exactly an optimal solution to $\delta V$-2EDSP, where $Q$ is a shortest $s t$-path with cost at most $\delta$ in $\hat{G}$, capturing the corresponding edge-disjoint path pair in the original graph $G$ share at most $\delta$ common vertices. Here we use $P \oplus Q$ to denote the set of edges $P \cup Q$ but with all opposite parallel edge pairs removed, where an opposite parallel edge pair is a pair of edges between an identical vertex pair but in opposite direction. The construction of the split-residual graph is similar to constructing a residual graph, except that every interior vertex on $P^{*}$ is split and the edges are modified accordingly.

Definition 5. (Residual graph) Given a graph $G$ with a weight function $w: E \rightarrow Z, P^{*}$ is a shortest st-path in $G$, the residual graph $\tilde{G}$ is constructed as follows. For each edge $(u, v) \in G \backslash P^{*}$, we add to $\tilde{G}$ an edge of the same weight as in $G$. For each edge $(u, v) \in P^{*}$, we add to $\tilde{G}$ a reversed edge $(v, u)$ with a weight $-w(e(u, v))$.

The main steps are as below. Firstly, construct a traditional residual graph $\tilde{G}:=G \cup \bar{P}^{*} \backslash P^{*}$, where $\bar{P}^{*}$ is the set of edges resulted from every edge on $P^{*}$ with direction reversed and weight set negative, i.e. for every edge $e=(u, v) \in P^{*}$, add $e^{\prime}=(v, u)$ to $\bar{P}^{*}$ with a weight $w\left(e^{\prime}\right)=-w(e)$. Secondly, split each interior vertex of $P^{*}$, say $v$, into two vertices $v_{1}$ and $v_{2}$, with an edge $\left(v_{1}, v_{2}\right)$ with weight 0 and cost 1 and an edge $\left(v_{2}, v_{1}\right)$ with weight 0 and cost 0 , where $Q$ going through edge $\left(v_{1}, v_{2}\right)$ indicates that in $G$ the corresponding paths resulting from $P^{*} \oplus Q$ will share the vertex $v$. Then redirect the edges that containing $v$ : (1) replace each edge $(v, u)$ in $\bar{P}^{*}$ by $\left(v_{1}, u_{2}\right) ;(2)$ replace each edge $(x, u) \notin \bar{P}^{*}$ with $u \in \bar{P}^{*}$ by $\left(x, u_{1}\right)$; (3) replace each edge $(v, y) \notin \bar{P}^{*}$ with $v \in \bar{P}^{*}$ by $\left(v_{2}, y\right)$. Moreover, the redirected edges are with cost 0 and the same weight as the original edges. Note that the above splitting can also deal with edge $(x, y)$ with either $x \in \bar{P}^{*}$ or $y \in \bar{P}^{*}$. For briefness, we denote by $E_{s}$ the set of cost 1 edges.

The detailed construction is formally as in Algorithm 1 (An example of splitting vertices is depicted as in Figure 2).

Proposition 6. Let $\left\{P_{1}^{*}, P_{2}^{*}\right\}$ be a spiral optimal solution to $\delta V$-2EDSP wrt $G$ and $P^{*}$. Let $H=P_{1}^{*} \cup P_{2}^{*} \cup P^{*}, \hat{H}$ be the split residual graph for $H$ wrt $P^{*}$. Assume that $E_{s} \subseteq \hat{H}$ 
is the set of edges between the pairs of split vertices for each $v \in P^{*}$. Then there must exist in $\hat{H}$ an st-path $Q$, for which $c(Q) \leq \delta$ and $w\left(P^{*}\right)+w(Q) \leq w\left(P_{1}^{*}\right)+w\left(P_{2}^{*}\right)$ both hold.

Proof. Following Lemma 4, the set of common interior vertices of $P_{1}^{*}$ and $P_{2}^{*}$, say $V\left(P_{1}^{*} \cap P_{2}^{*}\right) \backslash\{s, t\}=$ $\left\{v^{1}, v^{2}, \cdots, v^{\delta}\right\}$, must be all on the shortest path $P^{*}$. Then $P_{1}^{*}\left(v^{h}, v^{h+1}\right)$ and $P_{2}^{*}\left(v^{h}, v^{h+1}\right), h=0, \ldots, \delta$, is a pair of vertex disjoint paths between $v^{h}$ and $v^{h+1}$ in $H$. We will show that there exists a path $Q^{h} \subseteq \hat{H}$ between $v_{2}^{h}$ and $v_{1}^{h+1}$ in $\hat{H}$ with only edges of cost 0 . Then there exists an st-path $Q=s Q^{0} v_{1}^{1} v_{2}^{1} Q^{1} v_{1}^{2} \ldots v_{1}^{h} v_{2}^{h} Q^{h} v_{1}^{h+1} \ldots v_{1}^{\delta} v_{2}^{\delta} Q^{\delta} t$, whose total cost is $\delta$ as edge $\left(v_{1}^{h}, v_{2}^{h}\right), h=1, \ldots, \delta$, is with cost 1 while every other edge on the path is with cost 0 .

It remains only to construct $Q^{h}, \forall h$. We denote the set of maximal segments in $P^{*} \cap P_{i}^{*}(i=1,2)$ by $\mathcal{S}_{i}=$ $\left\{P^{*}\left(x_{i, j}, y_{i, j}\right) \mid j=1, \ldots, h_{i}\right\}$. W.l.o.g. assume that the first and the last maximal segments both belong to $P_{2}^{*}$, i.e. $x_{2, j}=v_{2}^{h}$ and $y_{2, h_{1}}=v_{1}^{h+1}$. Note that $\left(x_{2, j}, y_{2, j}\right)$ is exactly the previous maximal segment before $\left(x_{1, j}, y_{1, j}\right)$. Then we can construct $Q^{h}$ by using edges in $\hat{H}$ only:

$$
\begin{aligned}
& Q^{h}=\bigcup_{j=1}^{h_{1}}\left[\bar{P}^{*}\left(x_{1, j}, y_{2, j}\right) \cup P_{2}^{*}\left(y_{2, j}, x_{2, j+1}\right)\right. \\
& \left.\cup \bar{P}^{*}\left(x_{2, j+1}, y_{1, j}\right) \cup P_{1}^{*}\left(y_{1, j}, x_{1, j+1}\right)\right] \cup P_{1}^{*}\left(v_{2}^{h}, x_{1, j}\right)
\end{aligned}
$$

where $x_{1, h+1}=t$. Because the constructed $Q^{h}$ contains only segments of $P_{1}^{*} \cup P_{2}^{*}$ and $\bar{P}^{*}$ whose edges are of cost $0, Q$ is an st-path in $\hat{H}$ of cost at most $\delta$. Besides, $\bigcup_{j=1}^{h_{1}}\left[\bar{P}^{*}\left(x_{1, j}, y_{2, j}\right) \cup \bar{P}^{*}\left(x_{2, j+1}, y_{1, j}\right)\right]$ are exactly the set of edges resulting from reversing edges of $P^{*} \backslash\left(\mathcal{S}_{1} \cup \mathcal{S}_{2}\right)$. Therefore, we have $w\left(P^{*}\right)+w(Q)=w\left(\mathcal{S}_{1}\right)+w\left(\mathcal{S}_{2}\right)+$ $w\left(P_{1}^{*} \backslash \mathcal{S}_{1}\right)+w\left(P_{2}^{*} \backslash \mathcal{S}_{2}\right)=w\left(P_{1}^{*}\right)+w\left(P_{2}^{*}\right)$. This completes the proof.

Lemma 7. Let $Q$ be a shortest st-path with cost bounded by $\delta$ in $\hat{G}$ wrt $G$ and $P^{*}$, where $P^{*}$ is a shortest st-path in $G$. Assume that $Q^{\prime}$ is $Q$ except contracting every edge between each pair of split vertices. Then $P^{*} \oplus Q^{\prime}$ is exactly the union of two edge disjoint paths between $s$ and $t$, which have a minimum total weight and share at most $\delta$ common vertices.

Proof. We will firstly show that $P^{*} \oplus Q^{\prime}$ is exactly composed by two edge disjoint $s t$-paths of $G$, which shares at most $\delta$ common vertices; secondly that $w\left(P^{*} \oplus Q^{\prime}\right) \leq w(O P T)$, where $O P T$ is an optimal solution to $\delta V$-2EDSP.

For the first, let $Q^{\prime}$ be $Q$ except contracting every edge between every pair of split vertices. We need only to show the edges of $P^{*} \oplus Q^{\prime}$ exactly compose two edge disjoint $s t$-paths in $G$. First, $P^{*} \cup Q^{\prime}$ contains an $s t$-flow of value 2 , since $s$ and $t$ are respectively with a degree 2 and -2 while every other vertex of $P^{*} \cup Q^{\prime} \backslash\{s, t\}$ is with degree 0 . Then because $P^{*} \oplus$ $Q^{\prime}$ is $P^{*} \cup Q^{\prime}$ but removing all parallel edge pairs, $P^{*} \oplus Q^{\prime}$ contains only edges in $G$. Moreover, each removed parallel edge pair is actually a cycle where each vertex is of degree 0 , so in $P^{*} \oplus Q^{\prime}, s$ and $t$ respectively remain degree 2 and

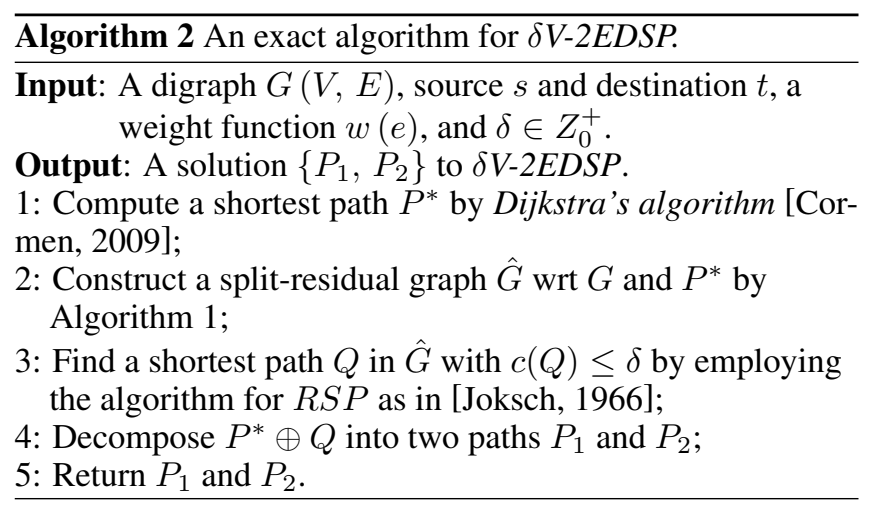

-2 , while every other vertex remains degree 0 . Therefore, $P^{*} \oplus Q^{\prime}$ is an $s t$-flow of value 2 , and hence is a pair of edge disjoint st-path in $G$ because each edge in $P^{*} \oplus Q^{\prime}$ is integral.

For the common vertices, let $\left\{P_{1}, P_{2}\right\}=P^{*} \oplus Q^{\prime}$ be the pair of disjoint st-paths. Assume that $v$ is an interior vertex shared by $P_{1}$ and $P_{2}$, i.e. $v \in P_{1} \cap P_{2} \backslash\{s, t\}$. Then following the construction of the auxiliary graph as in Algorithm $1, Q$ has to go through the edge from $v_{1}$ to $v_{2}$ for the split vertex $v$ in $\hat{G}$. Since $\left(v_{1}, v_{2}\right)$ is with cost 1 and every other edge is with cost $0, Q$ can at most go through $\delta$ such edges between split vertex pair as it has a cost no larger than $\delta$. Therefore, $P_{1}$ and $P_{2}$ share at most $\delta$ common vertices.

For the second, from Theorem 3, there must exist a spiral optimal solution to $\delta V$-2EDSP, say $\left\{P_{1}^{*}, P_{2}^{*}\right\}$. Let $H=P_{1}^{*} \cup$ $P_{2}^{*} \cup P^{*}$ and $\hat{H}$ be the accordingly residual graph wrt $P^{*}$. Then from Proposition 6, we can obtain an st-path $Q^{\prime \prime}$ in $\hat{H}$ with $c\left(Q^{\prime \prime}\right) \leq \delta$ and $w\left(P^{*}\right)+w\left(Q^{\prime \prime}\right) \leq w\left(P_{1}^{*}\right)+w\left(P_{2}^{*}\right)$. Since $\hat{H} \subseteq \hat{G}, Q^{\prime \prime}$ is also an st-path in $\hat{G}$ with both $c\left(Q^{\prime \prime}\right) \leq$ $\delta$ and $w\left(\bar{P}^{*}\right)+w\left(Q^{\prime \prime}\right) \leq w\left(P_{1}^{*}\right)+w\left(P_{2}^{*}\right)=w(O P T)$. Then because $Q$ is the shortest path in $\hat{G}$, we have $w(Q) \leq w\left(Q^{\prime \prime}\right)$, and hence $w\left(P^{*}\right)+w(Q) \leq w(O P T)$ holds. This completes the proof.

Following the above construction, we can obtain a splitresidual graph $\hat{G}=(\hat{V}, \hat{E})$, where the $\delta V-2 E D S P$ problem is transformed to the problem of finding a minimum weight st-path with cost bounded by $\delta$, namely the Binary Restricted Shortest Path $(B R S P)$ problem, which is clearly a special case of the restricted shortest path $(R S P)$ problem. Following the algorithm for RSP [Joksch, 1966], the BRSP problem can be solved in time $O(\delta|E|)$ by employing the dynamic programming method. Thus, $Q$ can be computed within time $O(\delta|E|)$. Then following Lemma 7, $P^{*} \oplus Q^{\prime}$ exactly composes an optimal solution to $\delta V-2 E D S P$, where $Q^{\prime}$ is $Q$ contracting each pair of split vertices back to one vertex in $G$, say contracting $v_{1}$ and $v_{2}$ to $v$. The formal layout of the whole algorithm is as in Algorithm 2.

Lemma 8. Algorithm 2 runs in time $O(\delta m+n \log n)$.

Proof. Let $m=|E(G)|$ and $n=|V(G)|$. Then, the algorithm takes $O(m+n \log n)$ time to run Dijkstra's algorithm against the original graph $G$ in Step 1 [Cormen, 2009], and $O(m+n)$ time to construct a split- residual graph $\hat{G}$ in Step 2 . In Step 3, the algorithm for BRSP runs in $O(m \delta)$, where $\delta$ is 
the bound of the number of common vertices. Other steps obviously takes trivial time comparing to the above ones. Therefore, the total runtime of Algorithm 2 is $O(\delta m+n \log n)$.

Combining Lemma 7 and 8, we immediately have the following theorem:

Theorem 9. Algorithm 2 runs in time $O(\delta m+n \log n)$ and outputs an optimal solution to $\delta V-2 E D S P$.

\section{Experimental Results}

In this section, we shall first evaluate the practical performance of Algorithm 2 (the exact algorithm, denoted as EA) by comparing its runtime and solution quality with another two algorithm baselines: the best previous algorithm (PA) presented in [Yallouz et al., 2016], and an exact algorithm via solving a flow integer linear program (ILP) formulation ${ }^{1}$ we designed for $\delta V-2 E D S P$. Then we show EA is scalable by running it against real-world network data including communication networks and P2P networks from Stanford Large Network Dataset Collection ${ }^{2}$ within Stanford Network Analysis Project (SNAP). These algorithms are implemented in Python 2.7 (The code is available upon request), on a PC with Intel Core i5 processor, and 8GB memory. Our implementation adopts the NetworkX library ${ }^{3}$ to construct and process graphs, the GLPK library ${ }^{4}$ to solve the ILP.

\subsection{Simulation Results}

In this subsection, we evaluate the runtime of the algorithms by simulation experiments, in which we generate a random graph from NetworkX with $n$ vertices, and $m$ edges each with a weight uniformly distributed in $[1,100]$. The number of allowed common vertices is the parameter $\delta$.

Simulation results comparing runtimes of EA, PA and ILP in seconds are depicted in Table 1. For each row of the table, we first randomly generated 1000 different graphs of the given size $(n, m)$, then respectively ran the three algorithms against each graph given a randomly chosen source and destination node pair, and at last computed the average time over the 1000 runs. Notice that during runs, the encountered infeasible graph instances do not contribute to our average time calculations. In the experiments, when the problem size is not larger than $n=100$ and $m=1000$, the runtime of EA is close to that of ILP ${ }^{5}$, but significantly faster than PA, say, about a thousand times faster. This matches the theoretical runtime difference between EA and PA, i.e. $O(\delta m+n \log n)$ vs $O\left(m n^{2}+n^{3} \log n\right)$. When the problem size grows larger, PA quickly becomes not scalable as it takes too long to produce a solution. So for larger graphs we only compared the runtimes of EA and ILP. The simulation results show that EA

\footnotetext{
${ }^{1}$ The formulation is available upon request but was omitted here due to space limit.

${ }^{2}$ https://snap.stanford.edu/data/

${ }^{3}$ https://networkx.github.io/

${ }^{4}$ https://www.gnu.org/software/glpk/

${ }^{5}$ Most likely because the random graph structure with a small $n$ becomes easier for the solution search of ILP solver that usually runs in worst case exponential time in $n$.
}

\begin{tabular}{|c|l|l|l||l|l|l|}
\hline Size $(n, m, \delta)$ & EA $(\mathrm{s})$ & PA $(\mathrm{s})$ & ILP $(\mathrm{s})$ & Size $(n, m, \delta)$ & EA $(\mathrm{s})$ & ILP $(\mathrm{s})$ \\
\hline \hline$(50,250,5)$ & 0.023 & 7.843 & 0.029 & $(500,25000,10)$ & 0.720 & 1.067 \\
\hline$(55,302,6)$ & 0.026 & 10.404 & 0.031 & $(550,30250,10)$ & 1.0923 & 1.476 \\
\hline$(60,5360,6)$ & 0.03 & 14.515 & 0.032 & $(600,36000,10)$ & 1.240 & 1.871 \\
\hline$(65,422,6)$ & 0.033 & 20.381 & 0.034 & $(650,42250,10)$ & 1.344 & 2.552 \\
\hline$(70,490,6)$ & 0.035 & 26.468 & 0.035 & $(700,49000,10)$ & 1.536 & 3.392 \\
\hline$(75,562,8)$ & 0.041 & 34.255 & 0.047 & $(750,56250,10)$ & 1.603 & 3.554 \\
\hline$(80,640,8)$ & 0.044 & 43.820 & 0.051 & $(800,64000,12)$ & 1.906 & 4.529 \\
\hline$(85,722,8)$ & 0.053 & 54.767 & 0.061 & $(850,72250,12)$ & 2.200 & 5.688 \\
\hline$(90,810,10)$ & 0.075 & 68.818 & 0.074 & $(900,81000,12)$ & 2.468 & 7.459 \\
\hline$(95,902,10)$ & 0.080 & 85.434 & 0.084 & $(950,90250,12)$ & 2.805 & 8.492 \\
\hline$(100,1000,10)$ & 0.090 & 104.518 & 0.095 & $(1000,100000,12)$ & 3.041 & 10.039 \\
\hline
\end{tabular}

Table 1: Runtime analysis on randomly generated graphs.

\begin{tabular}{|c|l|l|l||l|l|l|l|}
\hline Networks & \#Nodes & \#Edges & EA (s) & Networks & \#Nodes & \#Edges & EA (s) \\
\hline \hline Email-Enron & 36,692 & 183,831 & 20.76 & p2p-Gnut.09 & 8,114 & 26,013 & 2.40 \\
\hline Email-EuAll & 265,214 & 420,045 & 35.04 & p2p-Gnut.24 & 26,518 & 65,369 & 6.02 \\
\hline p2p-Gnut.05 & 8,846 & 31,839 & 2.55 & p2p-Gnut.25 & 22,687 & 54,705 & 5.13 \\
\hline p2p-Gnut.06 & 8,717 & 31,525 & 2.94 & p2p-Gnut.30 & 36,682 & 88,328 & 8.44 \\
\hline p2p-Gnut.08 & 6,301 & 20,777 & 1.92 & p2p-Gnut.31 & 62,586 & 147,892 & 13.40 \\
\hline
\end{tabular}

Table 2: Runtime analysis against network datasets from SNAP.

still runs much faster than ILP and more importantly the gap between these two grows with graph size.

It is also worth mentioning that, when testing against the same graph, the min-sum weights of the solutions, produced by all three algorithms, coincide in all experiments on the generated thousands of graphs. It was known that ILP and PA always output optimal solutions, so this observation can be regarded as an experimental evidence that our EA also produces optimal solutions, complementing our optimality proof.

\subsection{Real-world Evaluation}

Besides simulation results, we also ran EA against some realworld network datasets from the SNAP collections. In the experiment, we ran EA for 200 times and each time we instead randomly pick two vertices in the network as source and destination. We again report the average runtime for EA. We also reasonably set $\delta=10$, because in real networks, many protocols (TCP/IP etc.) only allow a data package pass through at most 16 routers from source to destination [Kurose and Ross, 2009]. Therefore a shortest path for transmitting data will have at most 16 interior vertices in most cases.

The experimental results depicted in Table 2 show that, roughly the runtime of EA is linearly dependent on the number of edges of the network. Moreover, EA takes up to $35 \mathrm{sec}-$ onds to process a network with $265 \mathrm{k}$ nodes and 420k edges whereas PA and ILP in our experiments can not even complete in many hours. Also noting that, although currently EA takes tens of seconds to process median size networks, it can be significantly accelerated with a more sophisticated $\mathrm{C} / \mathrm{C}++$ implementation and a higher end computing platform. These together will allow EA to efficiently process much larger graphs with hundreds of millions of nodes and edges. This is also supported from the time complexity of EA that is almost linear in the network size. 


\section{Conclusion}

In this paper, we carried on studying the problem of finding minimum-weight $k$ edge-disjoint partially vertex-disjoint paths, namely $\delta V-k E D S P$. We proposed an optimal algorithm for $\delta V-2 E D S P$ that efficiently runs in $O(\delta m+n \log n)$ time and in experiments demonstrates a significant runtime gain. This greatly improves the previous best runtime bound of $O\left(m n^{2}+n^{3} \log n\right)$ [Yallouz et al., 2016], where $m, n$ and $\delta$ are respectively the number of edges, vertices and allowed shared vertices. Note that the time complexity of our algorithm flexibly depends on $\delta$, which is at most $n$ (though a fixed $\delta$ is more practical), so the worst-case complexity is $O(m n+n \log n)-$ still a much better bound. We are currently investigating solving $\delta V-k E D S P$ by extending our technique for $\delta V-2 E D S P$. Also we are considering the counterpart problem $\delta E-k E D S P$, in which the number of edges shared by at least two paths is bounded by $\delta$ instead.

\section{Acknowledgements}

The research is supported by Natural Science Foundation of China (Nos. 61772005, 61300025) and Natural Science Foundation of Fujian Province (No. 2017J01753).

\section{References}

[Ahuja et al., 1993] Ravindra K Ahuja, Thomas L Magnanti, and James B Orlin. Network flows: theory, algorithms, and applications. 1993.

[Akiba et al., 2015] Takuya Akiba, Takanori Hayashi, Nozomi Nori, Yoichi Iwata, and Yuichi Yoshida. Efficient top-k shortest-path distance queries on large networks by pruned landmark labeling. In $A A A I$, volume 15 , pages $25-$ 30, 2015.

[Andrews et al., 2005] Matthew Andrews, Julia Chuzhoy, Sanjeev Khanna, and Lisa Zhang. Hardness of the undirected edge-disjoint paths problem with congestion. In Foundations of Computer Science, 2005. FOCS 2005. 46th Annual IEEE Symposium on, pages 226-241. IEEE, 2005.

[Andrews, 2010] Matthew Andrews. Approximation algorithms for the edge-disjoint paths problem via raecke decompositions. In Foundations of Computer Science (FOCS), 2010 51st Annual IEEE Symposium on, pages 277-286. IEEE, 2010.

[Chekuri and Ene, 2013] Chandra Chekuri and Alina Ene. Poly-logarithmic approximation for maximum node disjoint paths with constant congestion. In Proceedings of the twenty-fourth annual ACM-SIAM symposium on Discrete algorithms, pages 326-341. Society for Industrial and Applied Mathematics, 2013.

[Chekuri et al., 2005] Chandra Chekuri, Sanjeev Khanna, and F Bruce Shepherd. Multicommodity flow, well-linked terminals, and routing problems. In Proceedings of the thirty-seventh annual ACM symposium on Theory of computing, pages 183-192. ACM, 2005.

[Chuzhoy, 2016] Julia Chuzhoy. Routing in undirected graphs with constant congestion. SIAM Journal on Computing, 45(4):1490-1532, 2016.
[Cormen, 2009] Thomas H Cormen. Introduction to algorithms. MIT press, 2009.

[Garey and Johnson, 2002] Michael R Garey and David S Johnson. Computers and intractability, volume 29. wh freeman New York, 2002.

[Guo et al., 2015] Longkun Guo, Kewen Liao, Hong Shen, and Peng Li. Brief announcement: Efficient approximation algorithms for computing $\mathrm{k}$ disjoint restricted shortest paths. In Proceedings of the 27th ACM symposium on Parallelism in Algorithms and Architectures, pages 62-64. ACM, 2015.

[Hansen and Abdoulahi, 2016] Eric A Hansen and Ibrahim Abdoulahi. General error bounds in heuristic search algorithms for stochastic shortest path problems. In $A A A I$, pages 3130-3137, 2016.

[Hassin, 1992] Refael Hassin. Approximation schemes for the restricted shortest path problem. Mathematics of $O p$ erations research, 17(1):36-42, 1992.

[Imamichi et al., 2016] Takashi Imamichi, Takayuki Osogami, and Rudy Raymond. Truncating shortest path search for efficient map-matching. In IJCAI, pages 589$595,2016$.

[Joksch, 1966] Hans C Joksch. The shortest route problem with constraints. Journal of Mathematical analysis and applications, 14(2):191-197, 1966.

[Kurose and Ross, 2009] James F Kurose and Keith W Ross. Computer networking: a top-down approach, volume 4. Addison Wesley Boston, USA, 2009.

[Lorenz and Raz, 2001] Dean H Lorenz and Danny Raz. A simple efficient approximation scheme for the restricted shortest path problem. Operations Research Letters, 28(5):213-219, 2001.

[Orda and Sprintson, 2004] Ariel Orda and Alexander Sprintson. Efficient algorithms for computing disjoint qos paths. In INFOCOM 2004. Twenty-third AnnualJoint Conference of the IEEE Computer and Communications Societies, volume 1. IEEE, 2004.

[Raghavan and Tompson, 1987] Prabhakar Raghavan and Clark D Tompson. Randomized rounding: a technique for provably good algorithms and algorithmic proofs. Combinatorica, 7(4):365-374, 1987.

[Seymour and Kar, 2013] Zachary Seymour and Dulal Kar. Finding partially link-disjoint paths in wireless sensor networks. In Wireless Conference (EW), Proceedings of the 2013 19th European, pages 1-6. VDE, 2013.

[Suurballe and Tarjan, 1984] JW Suurballe and RE Tarjan. A quick method for finding shortest pairs of disjoint paths. Networks, 14(2):325-336, 1984.

[Suurballe, 1974] JW Suurballe. Disjoint paths in a network. Networks, 4(2):125-145, 1974.

[Yallouz et al., 2016] Jose Yallouz, Ori Rottenstreich, Peter Babarczi, Avi Mendelson, and Ariel Orda. Optimal linkdisjoint node-"somewhat disjoint" paths. In Network Protocols (ICNP), 2016 IEEE 24th International Conference on, pages 1-10. IEEE, 2016. 